\title{
Synthesis and Characterization of Graft Copolymer of Dextran and 2-Acrylamido-2-methylpropane Sulphonic Acid
}

\author{
Venkanna Azmeera, P. Adhikary, and S. Krishnamoorthi \\ Department of Chemistry, Banaras Hindu University, Varanasi 221005, India \\ Correspondence should be addressed to S. Krishnamoorthi, dr.skmoorthi@gmail.com \\ Received 21 May 2012; Accepted 24 July 2012 \\ Academic Editor: R. J. Linhardt
}

Copyright ( $) 2012$ Venkanna Azmeera et al. This is an open access article distributed under the Creative Commons Attribution License, which permits unrestricted use, distribution, and reproduction in any medium, provided the original work is properly cited.

A novel biodegradable graft copolymer of dextran (Dx) and 2-acrylamido-2-methyl-1-propane sulphonic acid (AMPS) was synthesized by grafting poly-AMPS chains onto dextran backbone by free radical polymerization using ceric ammonium nitrate (CAN) as an initiator. Different amounts of AMPS were used to synthesize four different grades of graft copolymers with different side chain lengths. These grafted polymers were characterized by elemental analysis, FTIR, ${ }^{1}$ HNMR, rheological technique, scanning electron microscopy (SEM), thermogravimetric analysis (TGA), and X-ray diffractometry (XRD). They exhibited efficient flocculation performance in kaolin suspension.

\section{Introduction}

Water soluble polymers have received a great attention in recent years. Carbohydrate polymers are often preferred over synthetic polymers due to their nontoxic, low-cost, ease of availability, and biodegradability characteristics [1-3]. They are used in pharmaceuticals, detergents, cosmetics, textiles production, and in waste-water treatment (flocculation) [4].

The choice of dextran relies on several aspects [5-8]. First, dextran of various molecular weights is commercially available. Second, the degree of branching of the dextran can be as low as $0.5 \%$, making them ideal molecular model for scientific studies. Third, dextran possesses high water solubility, is stable under mild acid and basic conditions, and contains a large number of hydroxyl groups for conjugation [9-11]. Dextran (Dx) $\left(\mathrm{C}_{6} \mathrm{H}_{10} \mathrm{O}_{5}\right)_{n}$ has been successfully used in the medical and biomedical fields for more than four decades. Dextran is the generic term for a family of neutral water soluble polysaccharides consisting (95\%) of $\alpha$ - $(1 \rightarrow 6)$ linked $D$-glucose main chain with varying branches, and $5 \%$ branches may arise from $\alpha-(1 \rightarrow 2), \alpha$ $(1 \rightarrow 3)$, and $\alpha-(1 \rightarrow 4)$ glycosidic linkages. It has common solubility in water and dipolar aprotic solvents [12]. It is biocompatible and biodegradable and can lead to biopolymers derivatives with balanced hydrophobic/hydrophilic character [13].

Among polymeric flocculants, the synthetic polymers can be tailor-made by controlling molecular weight, molecular weight distribution, the structure of polymers, and the nature and percentage of ionic groups. Thus, the synthetic polymers are very efficient flocculants [14-16]. It has been observed from rheological studies that grafting of shear degradable polymers onto the rigid polysaccharides backbone provides fairly shear stable systems [17].

In this paper, we aim to report the synthesis and characterization of graft copolymers based on poly 2-acylamido-2methyl-1-propane sulphonic acid (PAMPS) and dextran by ceric ammonium nitrate (CAN) induced solution polymerization technique [18-20]. Four grades of graft copolymers (Dx-g-PAMPS1 to Dx-g-PAMPS4) were synthesized by varying the amount of AMPS in the reaction feed [21]. The graft copolymers were characterized by elemental analysis, FTIR, FT- ${ }^{1} \mathrm{H}-\mathrm{NMR}$, rheological technique, scanning electron microscopy (SEM), thermal analysis (TGA), and X-ray diffractometry (XRD). The flocculation efficiencies of graft 
TABLE 1: Synthetic details of the graft copolymers of Dx and PAMPS.

\begin{tabular}{lcccccc}
\hline Serial number & Graft copolymers & Dextran $(\mathrm{g})$ & AMPS $(\mathrm{g})$ & CAN $(\mathrm{mg})$ & GE $(\%)$ & Intrinsic viscosity $(\mathrm{dL} / \mathrm{g})$ \\
\hline 1 & Dx-g-PAMPS1 & 1 & 5 & 20 & 75.66 & 13.5 \\
2 & Dx-g-PAMPS2 & 1 & 6 & 20 & 83.42 & 14.2 \\
3 & Dx-g-PAMPS3 & 1 & 7 & 20 & 96.56 & 15.3 \\
4 & Dx-g-PAMPS4 & 1 & 8 & 20 & 54.88 & 14.6 \\
\hline
\end{tabular}

Percentage of grafting efficiency $(\mathrm{GE})=$ weight of pure graft copolymer/weight of product mixture $\times 100$.

TABLE 2: Elemental analysis and flocculation performance of Dx and its graft copolymers.

\begin{tabular}{|c|c|c|c|c|c|}
\hline \multirow{2}{*}{ Polymer } & \multicolumn{3}{|c|}{ Elemental analysis } & \multicolumn{2}{|c|}{ Flocculation performance } \\
\hline & Carbon $(\%)$ & Hydrogen $(\%)$ & Nitrogen $(\%)$ & Optimum dose (ppm) & Turbidity (NTU) \\
\hline $\mathrm{Dx}$ & 38.7 & 6.2 & 0.36 & 4 & 96 \\
\hline Dx-g-PAMPS1 & 36.9 & 6.8 & 4.6 & 8 & 70 \\
\hline Dx-g-PAMPS2 & 38.9 & 6.9 & 4.7 & 10 & 51 \\
\hline Dx-g-PAMPS3 & 37.1 & 6.5 & 5.1 & 4 & 23 \\
\hline Dx-g-PAMPS4 & 38.7 & 6.9 & 4.5 & 16 & 24 \\
\hline
\end{tabular}

copolymers were estimated in kaolin suspension. It was observed that the graft copolymers are efficient flocculants.

\section{Experimental}

2.1. Materials. Dextran (average $M_{W}=482000 \mathrm{Da}$ ) was procured from Sigma-Aldrich chemicals, USA, 2-acrylamido2-methyl-1-propane sulphonic acid (AMPS) was procured from Sigma-Aldrich chemicals, Germany. CAN was procured from Loba Chemie, Mumbai. Acetone and hydroquinone were procured from E. Merck, Mumbai. Kaolin is a gift from Rajmahal Quartz-Sand, Kolkata, India.

2.2. Synthesis of Graft Copolymers. Four grades of graft copolymers (Dx-g-PAMPS1 to Dx-g-PAMPS4) of Dx and PAMPS were synthesized by Ce (IV) induced solution polymerization technique. In a $50 \mathrm{~mL}$ conical flask, $1 \mathrm{~g}$ of dextran was taken and it was dissolved in $25 \mathrm{~mL}$ of water and after a clear solution chuck it downs the amount of AMPS. The mixture was stirred continuously for $30 \mathrm{~min}$ under nitrogen atmosphere and heating the reaction mixture up to $50^{\circ} \mathrm{C}$, then $20 \mathrm{mg}$ of CAN was added. The reaction was carried out for 3 hrs until the solution become viscous. The graft copolymers were precipitated with acetone and washed 10 times by acetone for removal of unreacted AMPS. The resultant graft copolymer was dried under vacuum at $50^{\circ} \mathrm{C}$. The synthesis details of the graft copolymers are summarized in Table 1.

\subsection{Characterization}

2.3.1. Elemental Analysis. The elemental analysis of dextran and all the graft copolymers was performed by using CHN analyzer, CE-440 Elemental analyzer, Mexico. The estimated analysis of three different elements, carbon, hydrogen and nitrogen, was undertaken. The results are shown in Table 2.

2.3.2. Intrinsic Viscosity Measurement. Intrinsic viscosity measurements of the aqueous solution of all Dx and graft copolymers were carried out with the help of Ubbelohde viscometer (CS/S: 0.00386) $25 \pm 0.1^{\circ} \mathrm{C}$. Intrinsic viscosity of all the samples was determined from the point of intersection of two extrapolated (to zero concentration) plots, that is, inherent viscosity versus concentration $\left(\eta_{\text {inh }}\right.$ versus $\mathrm{C}$ ) and reduced viscosity versus concentration $\left(\eta_{\mathrm{red}}\right.$ versus $\mathrm{C}$ ). The standard method used to evaluate intrinsic viscosity has been described earlier [7]. The intrinsic viscosities of all the graft copolymers are reported in Table 1.

2.3.3. FTIR Spectroscopy. A Thermo Nicolet FT-IR spectrophotometer (Model JASCO FT-IR-5300) was used to record the IR spectra within the range of $4000-400 \mathrm{~cm}^{-1}$. The IR spectra (Figure 1) of dextran, PAMPS, and graft copolymer (Dx-g-PAMPS3) were recorded in solid state using a $\mathrm{KBr}$ pellet method.

2.3.4. ${ }^{1}$ H FTNMR Spectroscopy. ${ }^{1} \mathrm{H}$ NMR spectra (Figure 2) of samples were recorded by JEOL AL 300 FT-NMR spectrometer with sample solution in dimethyl sulphoxide- $\mathrm{d}_{6}$ (DMSO- $\mathrm{d}_{6}$ ) and tetramethylsilane (TMS) was used as an internal reference $[22,23]$.

2.3.5. Rheological Measurements. The rheological (viscosity versus shear rate) measurements (Figure 3) of aqueous solutions of dextran and graft copolymers (Dx-g-PAM1 to Dx-g-PAMPS4) were carried out using controlled stress Brookfield DV-III Ultra, programmable rheometer. A $2^{\circ}$ cone of diameter $4 \mathrm{~cm}$ and with truncation of $46 \mu \mathrm{m}$ was used for the measurements.

2.3.6. Scanning Electron Microscopy (SEM). For this study, dextran was used in powder form, whereas the PAMPS and graft copolymer (Dx-g-PAMPS3) were in the form of small granules (Figure 4). A HRSEM SUPRA 40, ZEISS (Germany) was used for the above study. 


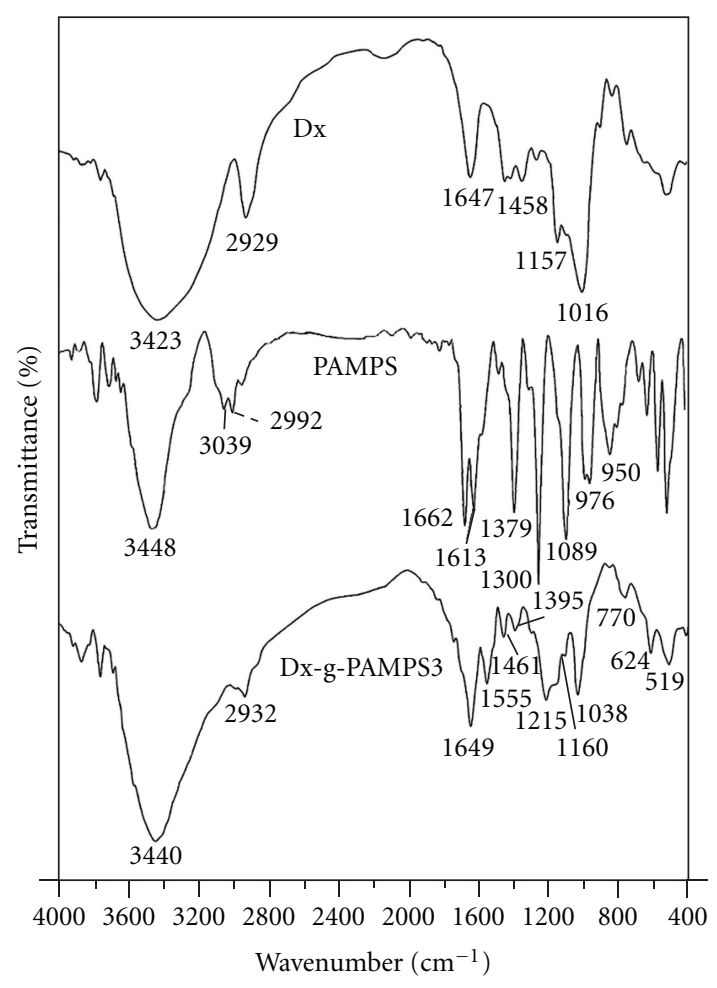

FIGURE 1: FT-IR spectra of Dx, PAMPS, and Dx-g-PAMPS3.

2.3.7. Thermal Analysis. The thermogravimetric analysis (TGA) of Dx, PAMPS, and its graft copolymer (Dx-gPAMPS3) was carried out with a TGA-DTA, METTERTOLEDO (Germany) instrument. Analysis of the samples (Figure 5) was performed up to a temperature of $600^{\circ} \mathrm{C}$, starting from $50^{\circ} \mathrm{C}$ in nitrogen atmosphere $[24,25]$. The heating rate was uniform in all cases at $10^{\circ} \mathrm{C}$ per minute.

2.3.8. X-Ray Diffractometry. Dextran, PAMPS, and the graft copolymer (Dx-g-PAMPS3) were subjected to XRD analysis (Figure 6). An 18-KW Cu-rotating anode Rigaku (Tokyo, Japan) X-ray powder diffractometer was used.

\section{Flocculation Study}

Jar test method was employed to estimate flocculation performance of synthesized graft copolymers. In $1 \mathrm{~L}$ beaker, $1 \mathrm{wt} \%$ kaolin suspension was taken. The beaker was placed on flocculator dipping the stirrer blade in the suspension. Under a low stirring condition, required quantity of polymer solution was added to beaker to make predetermined dose with respect of suspension volume. After the addition of polymer solution, the suspension was stirred at 185 RPM for $2 \mathrm{~min}$ followed by $100 \mathrm{RPM}$ for $5 \mathrm{~min}$. The flocs were allowed to settle down for 10 minutes. Clean supernatant liquid was drawn from a depth of $1.0 \mathrm{~cm}$ and its turbidity was measured using a digital nephelometer (digital nephelometer model, Bellstone Hitech International) to express the turbidity in nephelometric unit (NTU). A comparison of flocculation

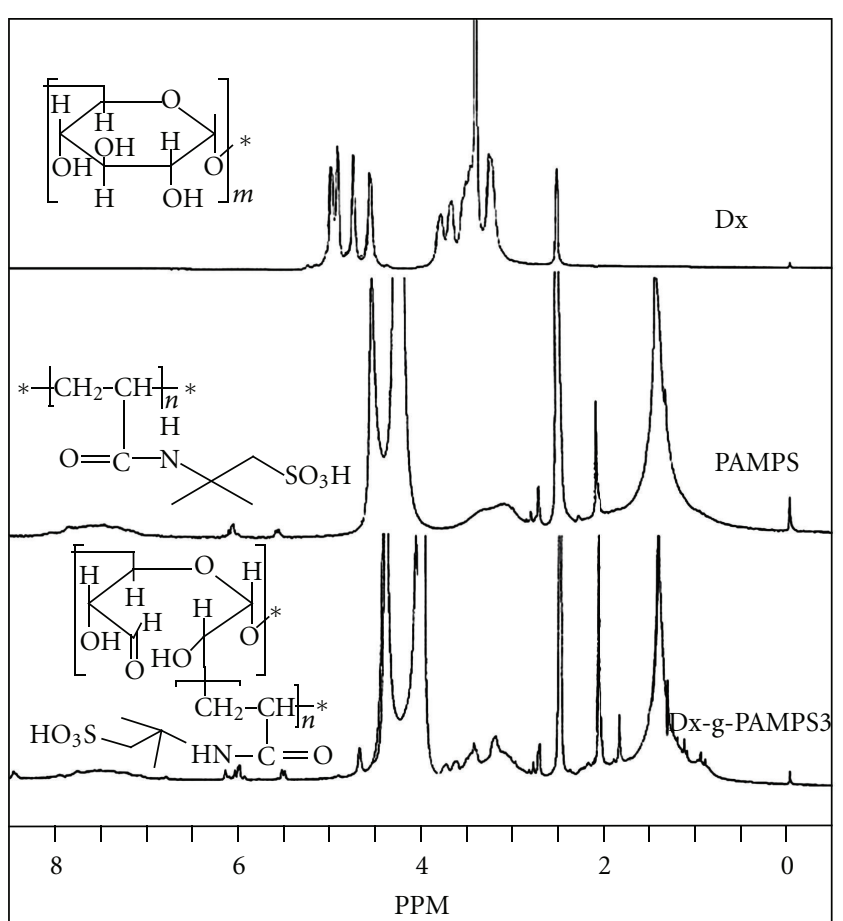

Figure 2: FT- ${ }^{1} \mathrm{H}$ NMR of Dx, PAMPS, and Dx-g-PAMPS3.

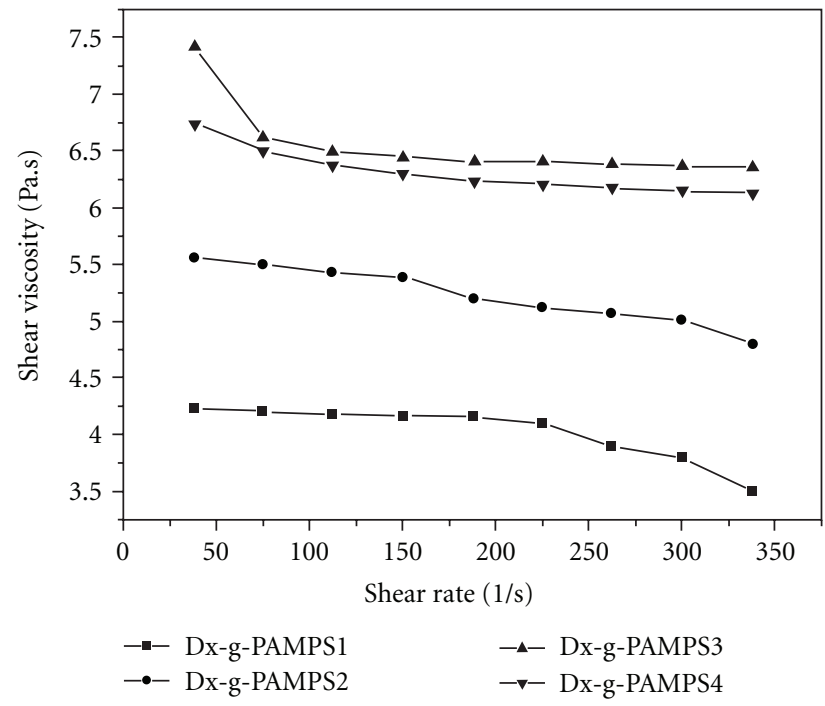

FIGURE 3: Viscosity versus shear rate curves of graft copolymers.

efficiency of Dx-g-PAMPS1 to Dx-g-PAMPS4 is shown in Table 2.

\section{Results and Discussion}

4.1. Synthesis of Graft Copolymers. Table 1 shows the synthetic details of the graft copolymers based on dextran and PAMPS by radical polymerization technique in aqueous medium using a ceric ammonium nitrate initiation system. The mechanism of ceric-ion-induced initiation involves the formation of chelate complex that decomposes to 


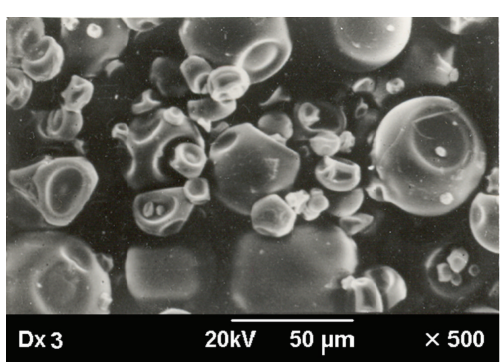

(a)

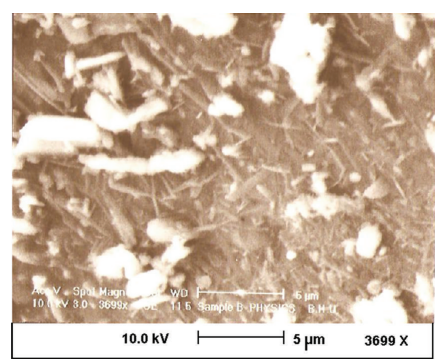

(b)

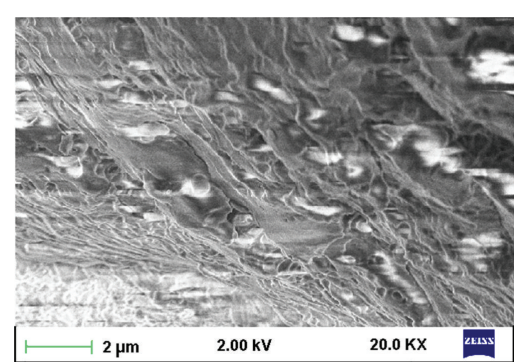

(c)

Figure 4: Scanning electron microscope of Dx, PAMPS, and Dx-g-PAMPS3.

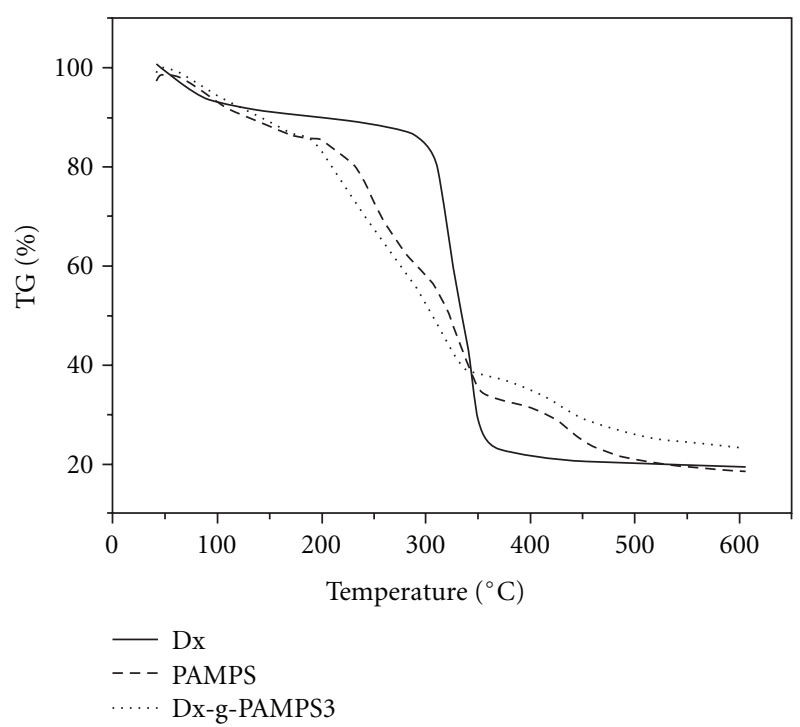

FIgure 5: Thermogravimetric analysis (TGA) of Dx, PAMPS, and Dx-g-PAMPS3.

generate free radical sites on the polysaccharide backbone. Four grades of graft copolymers (Dx-g-PAMPS1 to Dx-g-PAMPS4) were synthesized by varying the amount of 2-acrylamido-2-methyl-1-propane sulphonic acid. The amount of AMPS was varied in order to observe the effect with varying chain length of grafted PAMPS. At fixed concentration of CAN, fixed number of grafting sites on the polysaccharide backbone was expected. Thus, difference in AMPS concentration will lead to different chain length of PAMPS. The average length of PAMPS depends on the concentration of AMPS and dextran. At high concentration of AMPS, longer PAMPS chains were expected, whereas shorter chains were expected at lower concentration of AMPS. At optimum concentration of AMPS, longest PAMPS chains were obtained. Therefore, it was determined by intrinsic viscosity. In this case, Dx-g-PAMPS3 is the grade showing highest intrinsic viscosity of $15.3 \mathrm{dL} / \mathrm{g}$ having maximum chain length.

4.2. Elemental Analysis. The results of elemental analysis of Dx and its graft copolymers are given in Table 2. Dextran

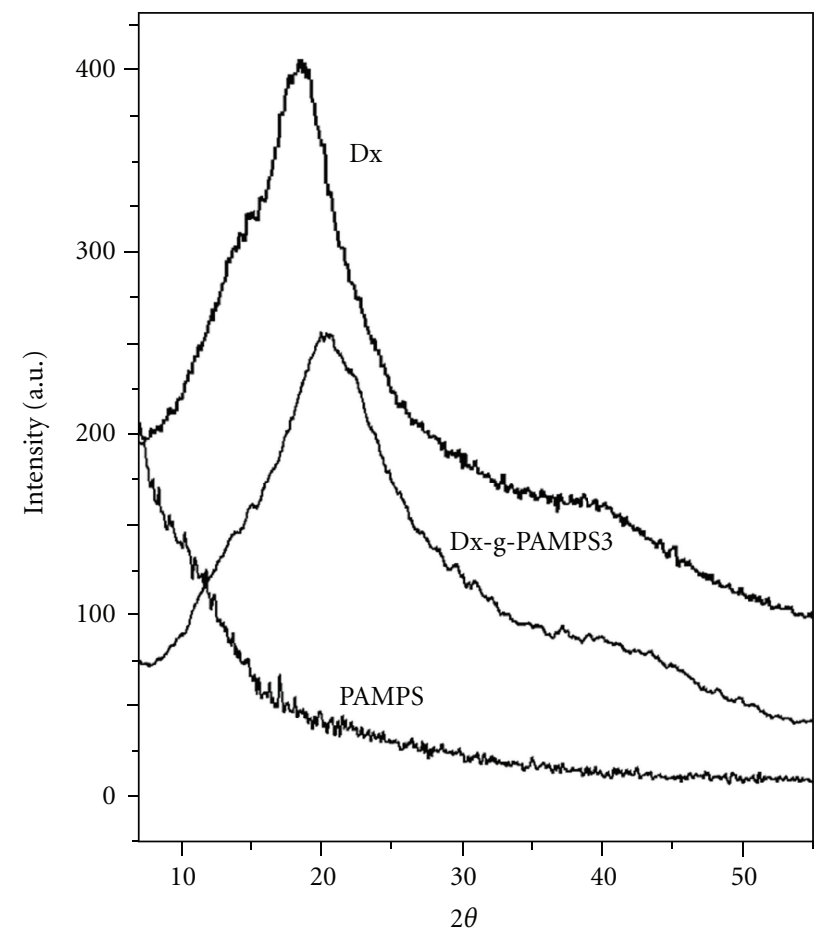

FIguRe 6: X-ray diffractometry of Dextran, PAMPS, and Dx-gPAMPS3.

does not show any significant presence of nitrogen. In case of graft copolymers, it was found that there is considerable percentage of nitrogen proving the grafting of PAMPS chains on the polysaccharides backbone. In the series of graft copolymers with $\mathrm{Dx}$, highest percentage of nitrogen was observed in case of Dx-g-PAMPS3. Since this graft copolymer showed highest intrinsic viscosity and maximum grafting efficiency (GE), the highest percentage of nitrogen can be explained on the basis of longer branches of PAMPS on the dextran backbone.

4.3. Intrinsic Viscosity Measurement. The intrinsic viscosity of a polymer is a measure of its hydrodynamic volume in solution, which in turn is a function of the polymer molecular weight, its structure, nature of solvent, and the temperature of the medium. Keeping the other factors 
constant, for two polymers of approximately similar molecular weight, a branched polymer will have lower hydrodynamic volume and hence a lower intrinsic viscosity as compared to its linear counterpart. Further, along a series of branched polymers, the longer the branches, the higher will be the intrinsic viscosity and vice versa. This has been observed in practice.

In the series of graft copolymers (Dx-g-PAMPS1 to Dx-gPAMPS4), the graft copolymer Dx-g-PAMPS3, having optimum AMPS concentration at a fixed CAN concentration, has higher intrinsic viscosity indicating that it has longer PAMPS chains. The intrinsic viscosity values of all graft copolymers are reported in Table 1.

4.4. FTIR Spectroscopy. FTIR spectra of the dextran and Dx-g-PAMPS3 are shown in Figure 1. The IR spectra of Dx, AMPS, and Dx-g-PAMPS3 are shown in Figure 2. In the IR spectrum of Dx, a broad peak present at $3423 \mathrm{~cm}^{-1}$ and is due to the stretching of $-\mathrm{OH}$. Due to hydrophilic nature of Dx, a peak shows at $1647 \mathrm{~cm}^{-1}$ is due to bending of water molecule. The peaks present at $2929 \mathrm{~cm}^{-1}$ and $1458 \mathrm{~cm}^{-1}$ represent the stretching and bending frequency of $-\mathrm{CH}_{2}$ group. A band at $1157 \mathrm{~cm}^{-1}$ confirms the presence of glycosidic linkage $(\mathrm{C}-\mathrm{O}-\mathrm{C})$ in dextran. The band in the region of $1016 \mathrm{~cm}^{-1}$ is due to the $\mathrm{C}-\mathrm{O}$ stretching. In case of AMPS spectrum, a broad band at $3448 \mathrm{~cm}^{-1}$ is due to the collapse of $-\mathrm{NH}$ and $-\mathrm{OH}\left(-\mathrm{SO}_{3} \mathrm{H}\right)$ stretching frequency. The peaks present at $3039 \mathrm{~cm}^{-1}$ and $2992 \mathrm{~cm}^{-1}$ represent the $\mathrm{C}-\mathrm{H}$ stretching frequency of $-\mathrm{CH}_{3}$ and $-\mathrm{CH}_{2}$, respectively. Two bands around 1662 and $1613 \mathrm{~cm}^{-1}$ are due to $\mathrm{C}=\mathrm{O}$ stretching (amide I band) and $\mathrm{N}-\mathrm{H}$ bending (amide II band), respectively. Two peaks at 1379 and $1300 \mathrm{~cm}^{-1}$ are due to asymmetric and symmetrical stretching of $(\mathrm{S}=\mathrm{O})$ group. The strong stretching absorption in the region of 1089 to $950 \mathrm{~cm}^{-1}$ represent S-O-C group. Dx-g-PAMPS3 shows absorption peaks at $3440 \mathrm{~cm}^{-1}(-\mathrm{NH}$ and $-\mathrm{OH})$, $2962 \mathrm{~cm}^{-1}$ (C-H stretching), $1649 \mathrm{~cm}^{-1}$ and $1555 \mathrm{~cm}^{-1}$ (amide I and amide II bands), $1461 \mathrm{~cm}^{-1}$ (C-H bending), 1395 and $1215 \mathrm{~cm}^{-1}$ (asymmetric and symmetric stretching of $\mathrm{S}=\mathrm{O}$ group), $1160 \mathrm{~cm}^{-1}$ (glycosidic linkage), and 1038 to $770 \mathrm{~cm}^{-1}$ (S-O-C group). The presence of all the peaks is due to that Dx and PAMPS are present in the graft copolymer (Dx-g-PAMPS3). This proved the grafting of AMPS on Dx backbone.

4.5. ${ }^{1} \mathrm{H}$ FTNMR Spectroscopy. Figure 2 shows the ${ }^{1} \mathrm{H}$ NMR of Dx, PAPMS, and Dx-g-PAMPS3 in DMSO solvent. In ${ }^{1} \mathrm{H}$ NMR of Dx signals in the region of $4 \mathrm{ppm}$ to $5 \mathrm{ppm}$ are due to the 3 and 6 -linked- $\alpha$-D-glc $p$ residues. The complex spectrum in the region of $3-4 \mathrm{ppm}$ resulted from protons in $\mathrm{C}_{2}, \mathrm{C}_{3}, \mathrm{C}_{4}, \mathrm{C}_{5}$, and $\mathrm{C}_{6}$. In the case of PAMPS, the resonance peaks are at $7.45 \mathrm{ppm}(-\mathrm{NH}-), 4.4 \mathrm{ppm}\left(-\mathrm{CH}_{2}-\right.$ $\left.\mathrm{SO}_{3} \mathrm{H}\right), 3.06 \mathrm{ppm}\left(-\mathrm{SO}_{3} \mathrm{H}\right), 2.70 \mathrm{ppm}$ (-CH-CO), $2.0 \mathrm{ppm}$ $\left(-\mathrm{CH}_{2}-\mathrm{CH}-\right)$, and $1.42 \mathrm{ppm}\left(-\mathrm{CH}_{3}\right)$. In the proton NMR of graft copolymer (Dx-g-PAMPS3), peaks were observed at $1.43 \mathrm{ppm}\left(-\mathrm{CH}_{3}\right), 1.85 \mathrm{ppm}\left(-\mathrm{CH}_{2}-\right), 2.71 \mathrm{ppm}(-\mathrm{CH}-\mathrm{CO})$, 3.2 to $3.7 \mathrm{ppm}$ (proton in $\mathrm{C}_{2}, \mathrm{C}_{3}, \mathrm{C}_{4}, \mathrm{C}_{5}$, and $\mathrm{C}_{6}$ ), $4.4 \mathrm{ppm}$ $\left(-\mathrm{CH}_{2}-\mathrm{SO}_{3} \mathrm{H}\right), 4.6 \mathrm{ppm}$ (anomeric proton), and $7.8 \mathrm{ppm}$
(-NH-). The resonance peaks of Dx segment and those of PAMPS repeating unit were also observed in case of graft copolymer. This proves the grafting of PAMPS on Dx backbone.

4.6. Rheological Studies. The rheological studies of aqueous solution of Dx-g-PAMPS1 to Dx-g-PAMPS4 were carried out at concentration of $0.5 \mathrm{wt} \%$. The shear viscosity versus shear rate on two axes in logarithmic scales was plotted. Figure 3 shows that the shear viscosity versus shear rate curves of graft copolymers of all the four graft copolymers in aqueous solution. In these cases, shear viscosity decreased with increase in shear rate and the samples showed shear thinning non-Newtonian behaviour (pseudoplastic). Among the four grades of graft copolymers, the Dx-g-PAMPS3 shows higher shear viscosity in the entire shear rate. This was due to the longer PAMPS chains grafted on to the dextran backbone.

4.7. Scanning Electron Microscopy. Figures 4(a), 4(b), and 4 (c) show the scanning electron micrographs of Dx, APMS, and Dx-g-PAMPS3, respectively. The surface morphology of Dx and PAMPS before grafting indicates spherical and small needle-like structures, respectively, which have been changed to fibrillar form after grafting (Dx-g-PAMPS3). The comparison of these figures reveals that grafting has taken place.

4.8. Thermal Analysis. The thermal stabilities of graft copolymers were investigated by thermogravimetric analysis (TGA) in a nitrogen stream at a heating rate of $20^{\circ} \mathrm{C} / \mathrm{min}$. The TGA curves of Dx, PAMPS, and Dx-g-PAMPS3 were studied in the range of $50^{\circ} \mathrm{C}-600^{\circ} \mathrm{C}$ (Figure 5). All the polymer samples exhibit three degradation stages. In the first stage, weight loss of Dx, PAMPS, and Dx-g-PAMPS3 was due to water evolving in the range of $50^{\circ} \mathrm{C}-150^{\circ} \mathrm{C}, 50^{\circ} \mathrm{C}-$ $230^{\circ} \mathrm{C}$, and $50^{\circ} \mathrm{C}-250^{\circ} \mathrm{C}$, respectively. In case of $\mathrm{Dx}$, the second decomposition temperature occurred at $300^{\circ} \mathrm{C}$ due to degradation of main chain. In case of AMPS and graft copolymer, the weight loss at $250^{\circ} \mathrm{C}$ was assigned to the decomposition of sulphonic acid groups. In the third stage, combustion of the degraded products began at $350^{\circ} \mathrm{C}$. In the last step of degradation of all the polymers, the graft copolymer has less weight loss than the other polymers. The thermal stability of Dx-g-PAMPS3 was higher than dextran and PAMPS beyond $350^{\circ} \mathrm{C}$. Before $350^{\circ} \mathrm{C}$, Dx-g-PAMPS3 was less stable than PAMPS and dextran.

4.9. X-Ray Diffractometry (XRD). XRD analysis of $\mathrm{Dx}$, PAMPS, and Dx-g-PAMPS3 is shown in Figure 6. Dx and graft copolymer do not show crystallinity in contrast to PAMPS which shows small crystallinity. This is indicative of absence of AMPS monomer in the graft copolymer.

\section{Flocculation Study}

The plots of supernatant turbidity versus graft polymer dosage for kaolin suspension are given in Table 2. It has 
been found that graft copolymer Dx-g-PAMPS3 shows better performance (lesser turbidity over lowest polymer dose) than other graft copolymers. This could be explained due to the fact that in graft copolymer, the dangling longer poly-PAMPS chains have better approachability to the contaminant.

\section{Conclusion}

From the results, it can be concluded that PAMPS is grafted on Dx. Variation in the synthetic parameters results in a series of graft copolymers with varying length of PAMPS chains. The FTIR and $\mathrm{FT}^{1} \mathrm{H}$ NMR spectra provide a strong proof of grafting. Rheological investigation shows that the graft copolymer having longer PAMPS chains has more shear viscosity. TGA results show that the graft copolymer has more thermal stability than corresponding Dx and PAMPS. Shear viscosity of the aqueous solution of the graft copolymer Dx-g-PAMPS3 is larger than the other graft copolymers. Morphology variation of Dx, PAMPS, and graft copolymers supports the above conclusion. X-ray diffraction pattern shows the amorphous nature of graft copolymer and absence of AMPS in the grafted product. Estimation of flocculation performance in kaolin suspension shows the graft copolymer Dx-g-PAMPS3 as the most effective flocculant. Thus, the graft copolymer in every way is more stable than the base polysaccharide. Thus, by grafting PAMPS onto dextran, shear stable efficient flocculants have been obtained and they can be used for the treatment of wastewater.

\section{Acknowledgment}

The authors are grateful to UGC (Grant number: 3757/2009), New Delhi, for the financial support.

\section{References}

[1] C. Nouvel, P. Dubois, E. Dellacherie, and J.-L. Six, "Controlled synthesis of amphiphilic biodegradable polylactide-grafted dextran copolymers," Journal of Polymer Science A, vol. 42, no. 11, pp. 2577-2588, 2004.

[2] T. Ouchi, T. Kontani, and Y. Ohya, "Mechanical property and biodegradability of solution-cast films prepared from amphiphilic polylactide-grafted dextran," Journal of Polymer Science A, vol. 41, no. 16, pp. 2462-2468, 2003.

[3] T. Ouchi, T. Kontani, R. Aoki, T. Saito, and Y. Ohya, "Characteristic properties of film prepared from poly(L-lactide)grafted dextran of a relatively high sugar unit content as a degradable biomaterial," Journal of Polymer Science A, vol. 44, no. 21, pp. 6402-6409, 2006.

[4] I. Uraz and A. Güner, "Comparison of molecular association of dextran and periodate-oxidized dextran in aqueous solutions," Carbohydrate Polymers, vol. 34, no. 3, pp. 127-130, 1997.

[5] H. Wondraczek, T. Elschner, and T. Heinze, "Synthesis of highly functionalized dextran alkyl carbonates showing nanosphere formation," Carbohydrate Polymers, vol. 83, no. 3, pp. 1112-1118, 2011.

[6] M. P. Bajgai, D. C. Parajuli, J. A. Ko, H. K. Kang, M.-S. Khil, and H. Y. Kim, "Synthesis, characterization and aqueous dispersion of dextran-g-poly(1,4-dioxan-2-one) copolymers," Carbohydrate Polymers, vol. 78, no. 4, pp. 833-840, 2009.

[7] S. Krishnamoorthi, D. Mal, and R. P. Singh, "Characterization of graft copolymer based on polyacrylamide and dextran," Carbohydrate Polymers, vol. 69, no. 2, pp. 371-377, 2007.

[8] B. G. De Geest, W. Van Camp, F. E. Du Prez, S. C. De Smedt, J. Demeester, and W. E. Hennink, "Biodegradable microcapsules designed via 'click' chemistry," Chemical Communications, no. 2, pp. 190-192, 2008.

[9] N. Pahimanolis, A.-H. Vesterinen, J. Rich, and J. Seppala, "Modification of dextran using click-chemistry approach in aqueous media," Carbohydrate Polymers, vol. 82, no. 1, pp. 78$82,2010$.

[10] M. Tang, H. Dou, and K. Sun, "One-step synthesis of dextranbased stable nanoparticles assisted by self-assembly," Polymer, vol. 47, no. 2, pp. 728-734, 2006.

[11] R. Y. Cheung, Y. Ying, A. M. Rauth, N. Marcon, and X. Y. $\mathrm{Wu}$, "Biodegradable dextran-based microspheres for delivery of anticancer drug mitomycin C," Biomaterials, vol. 26, no. 26, pp. 5375-5385, 2005.

[12] A. P. Rokhade, S. A. Patil, and T. M. Aminabhavi, "Synthesis and characterization of semi-interpenetrating polymer network microspheres of acrylamide grafted dextran and chitosan for controlled release of acyclovir," Carbohydrate Polymers, vol. 67, no. 4, pp. 605-613, 2007.

[13] J. Raynaud, B. Choquenet, E. Marie et al., "Emulsifying properties of biodegradable polylactide-grafted dextran copolymers," Biomacromolecules, vol. 9, no. 3, pp. 1014-1021, 2008.

[14] S. Krishnamoorthi, D. Mai, and R. P. Singh, "Characterization and solution properties of a partially hydrolyzed graft copolymer of polyacrylamide and dextran," Journal of Applied Polymer Science, vol. 110, no. 3, pp. 1297-1303, 2008.

[15] S. Krishnamoorthi, P. Adhikary, D. Mal, and R. P. Singh, "Novel polymeric flocculants based on polyacrylamide grafted dextran in kaolin suspension," Journal of Applied Polymer Science, vol. 118, no. 6, pp. 3539-3544, 2010.

[16] P. Adhikary, K. N. Tiwari, and R. P. Singh, "Synthesis, characterization, and flocculation characteristics of polyacrylamidegrafted glycogen," Journal of Applied Polymer Science, vol. 103, no. 2, pp. 773-778, 2007.

[17] Y. H. Gad, "Preparation and characterization of poly(2acrylamido-2-methylpropane- sulfonic acid)/Chitosan hydrogel using gamma irradiation and its application in wastewater treatment," Radiation Physics and Chemistry, vol. 77, no. 9, pp. 1101-1107, 2008.

[18] C. Zhang and A. J. Easteal, "Study of free-radical copolymerization of $\mathrm{N}$-isopropylacrylamide with 2-acrylamido-2methyl-1-propanesulphonic acid," Journal of Applied Polymer Science, vol. 88, no. 11, pp. 2563-2569, 2003.

[19] S. J. De Jong, S. C. De Smedt, M. W. C. Wahls, J. Demeester, J. J. Kettenes-van den Bosch, and W. E. Hennink, "Novel selfassembled hydrogels by stereocomplex formation in aqueous solution of enantiomeric lactic acid oligomers grafted to dextran," Macromolecules, vol. 33, no. 10, pp. 3680-3686, 2000.

[20] C. Perrino, S. Lee, S. W. Choi, A. Maruyama, and N. D. Spencer, "A biomimetic alternative to poly(ethylene glycol) as an antifouling coating: resistance to nonspecific protein adsorption of poly(L-lysine)-graft- dextran," Langmuir, vol. 24, no. 16, pp. 8850-8856, 2008.

[21] Y. Bao, J. Ma, and N. Li, "Synthesis and swelling behaviors of sodium carboxymethyl cellulose-g-poly(AA-co-AM-coAMPS)/MMT superabsorbent hydrogel," Carbohydrate Polymers, vol. 84, no. 1, pp. 76-82, 2011. 
[22] A. P. Goodwin, S. M. Tabakman, K. Welsher, S. P. Sherlock, G. Prencipe, and H. Dai, "Phospholipid-dextran with a single coupling point: a useful amphiphile for functionalization of nanomaterials," Journal of the American Chemical Society, vol. 131, no. 1, pp. 289-296, 2009.

[23] A. Richard, A. Barras, A. B. Younes, N. Monfilliette-Dupont, and P. Melnyk, "Minimal chemical modification of reductive end of dextran to produce an amphiphilic polysaccharide able to incorporate onto lipid nanocapsules," Bioconjugate Chemistry, vol. 19, no. 7, pp. 1491-1495, 2008.

[24] J. Maia, R. A. Carvalho, J. F. J. Coelho, P. N. Simões, and M. H. Gil, "Insight on the periodate oxidation of dextran and its structural vicissitudes," Polymer, vol. 52, no. 2, pp. 258-265, 2011.

[25] E. Fathi, N. Atyabi, M. Imani, and Z. Alinejad, "Physically crosslinked polyvinyl alcohol-dextran blend xerogels: morphology and thermal behavior," Carbohydrate Polymers, vol. 84, no. 1, pp. 145-152, 2011. 


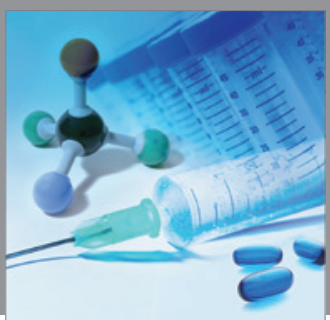

International Journal of

Medicinal Chemistry

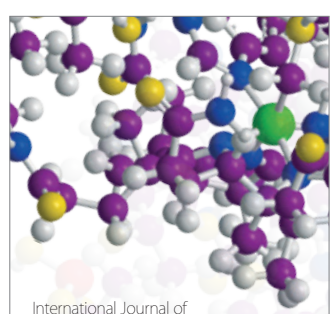

Carbohydrate Chemistry

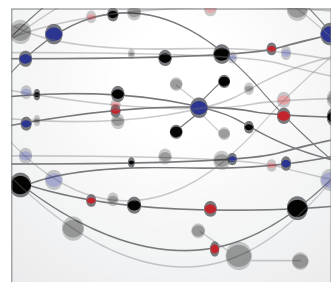

The Scientific World Journal
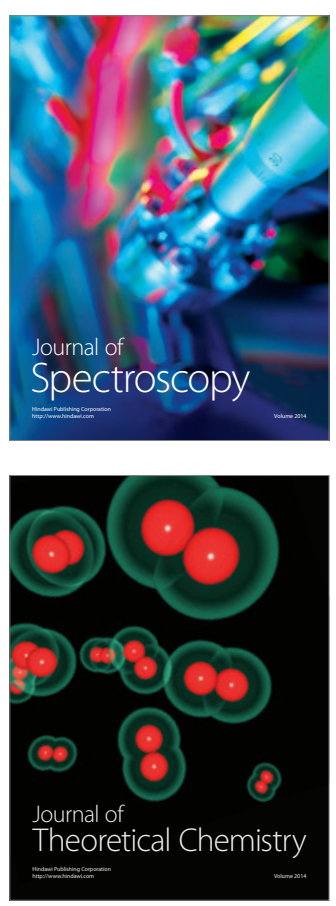
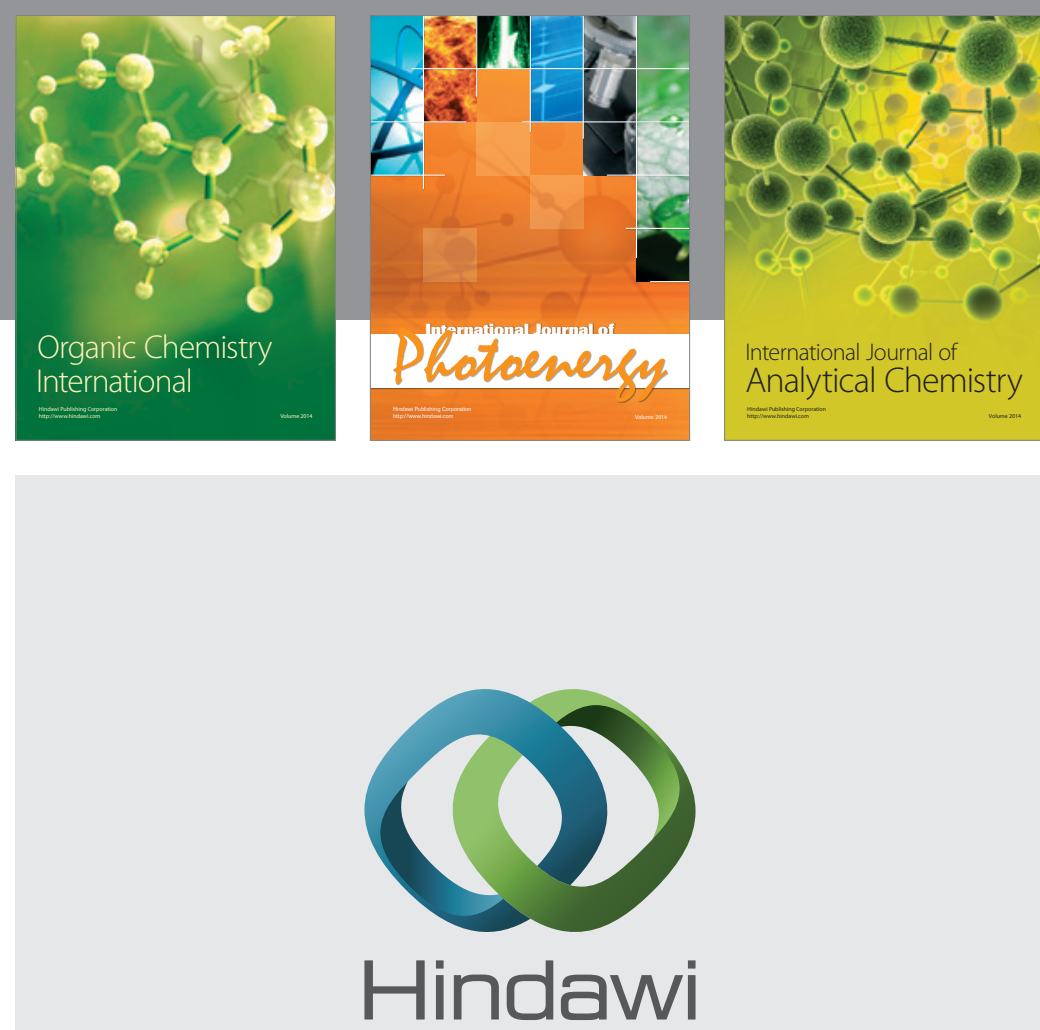

Submit your manuscripts at

http://www.hindawi.com
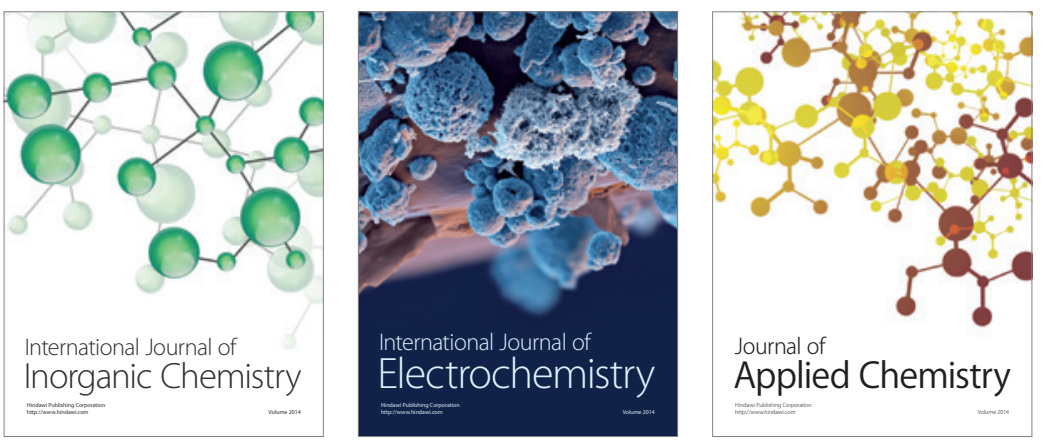

Journal of

Applied Chemistry
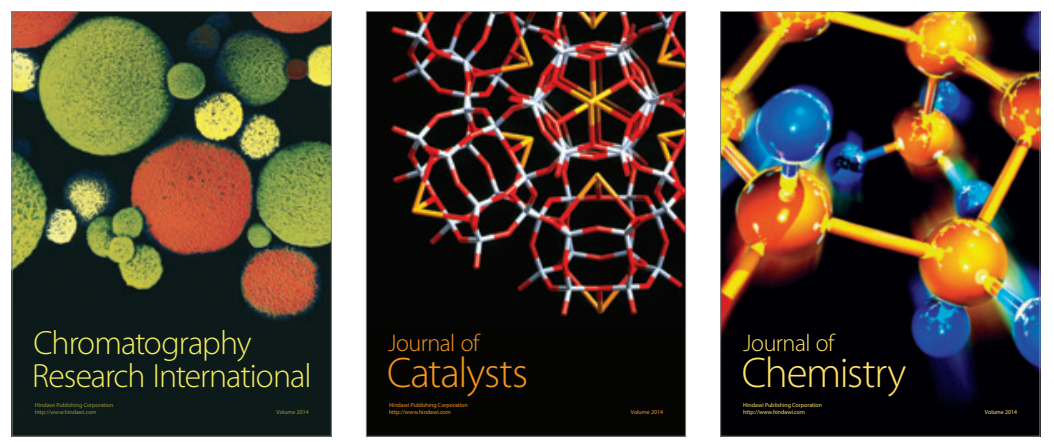
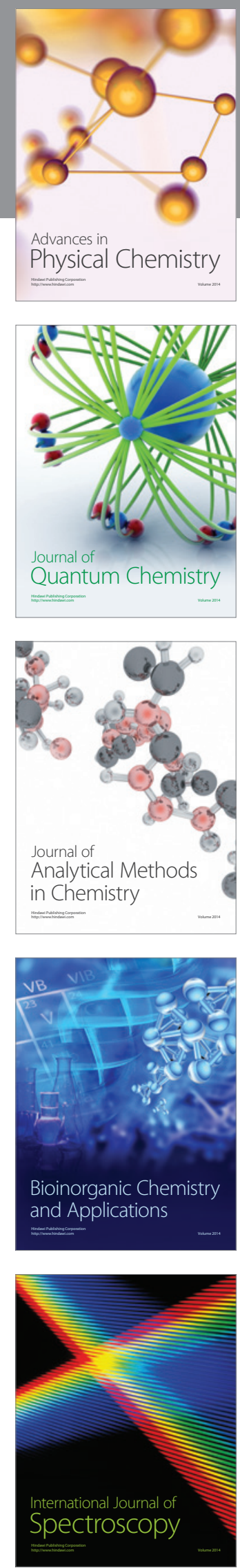